\title{
The Role of Anterior Cingulate Cortex in the Affective Evaluation of Conflict
}

\author{
Senne Braem ${ }^{1}$ Joseph A. King ${ }^{2}$, Franziska M. Korb ${ }^{2}$, Ruth M. Krebs ${ }^{1}$, \\ Wim Notebaert ${ }^{1}$, and Tobias Egner ${ }^{2}$
}

\begin{abstract}
An influential theory of ACC function argues that this brain region plays a crucial role in the affective evaluation of performance monitoring and control demands. Specifically, controldemanding processes such as response conflict are thought to be registered as aversive signals by ACC, which in turn triggers processing adjustments to support avoidance learning. In support of conflict being treated as an aversive event, recent behavioral studies demonstrated that incongruent (i.e., conflict inducing), relative to congruent, stimuli can speed up subsequent negative, relative to positive, affective picture processing. Here, we used fMRI to investigate directly whether ACC
\end{abstract}

\section{INTRODUCTION}

ACC has been implicated in a variety of psychological processes such as emotion regulation (Etkin, Egner, \& Kalisch, 2011), pain perception (Tracey \& Mantyh, 2007), and cognitive control (Ridderinkhof, Ullsperger, Crone, \& Nieuwenhuis, 2004). In an integrative review on ACC, Shackman and colleagues (2011) concluded that its domain-general role must be one of driving aversively motivated behavior. Consistent with this proposal, Botvinick (2007) suggested that ACC generally signals suboptimal outcomes that drive avoidance learning. In doing so, Botvinick (2007) reinterpreted ACC's role in one of its most studied cognitive control functions: the monitoring of cognitive conflict (Botvinick, Cohen, \& Carter, 2004; Botvinick, Braver, Barch, Carter, \& Cohen, 2001). Namely, Botvinick (2007) hypothesized that ACC's response to cognitive conflict (i.e., the simultaneous activation of mutually incompatible stimulus, task, or response representations) is registered as an aversive signal, much like pain or punishment. Although this idea has gained widespread attention and inspired many behavioral studies to test if conflict is indeed experienced as an aversive event (for reviews, see Dreisbach \& Fischer, 2015; Inzlicht, Bartholow, \& Hirsh, 2015; van Steenbergen, 2015), to the best of our knowledge, no imaging study to date has investigated directly whether ACC's response to cognitive conflict does in fact also register as an affec-

${ }^{1}$ Ghent University, ${ }^{2}$ Duke University activity in response to negative versus positive pictures is modulated by preceding control demands, consisting of conflict and task-switching conditions. The results show that negative, relative to positive, pictures elicited higher ACC activation after congruent, relative to incongruent, trials, suggesting that ACC's response to negative (positive) pictures was indeed affectively primed by incongruent (congruent) trials. Interestingly, this pattern of results was observed on task repetitions but disappeared on task alternations. This study supports the proposal that conflict induces negative affect and is the first to show that this affective signal is reflected in ACC activation.

tively aversive signal (Botvinick, 2007; see also Shenhav, Botvinick, \& Cohen, 2013).

In the wake of Botvinick's (2007) proposal, a growing number of behavioral studies confirmed its first assumption, namely, that cognitive conflicts appear to be experienced as aversive events (Dignath \& Eder, 2015; Fritz \& Dreisbach, 2013, 2015; Schouppe et al., 2012, 2015; Dreisbach \& Fischer, 2012; Brouillet, Ferrier, Grosselin, \& Brouillet, 2011; for a review, see Dreisbach \& Fischer, 2015). For example, Dreisbach and Fischer (2012) investigated this by combining a classic color Stroop task (Stroop, 1935) with an affective priming paradigm (Fazio, 2001). In an affective priming paradigm, participants are asked to categorize affective words (e.g., "flower" or "bomb") based on their valence (i.e., positive or negative) as fast as possible. These affective probes are preceded by task-irrelevant stimuli (i.e., the prime). If the prime elicits an affective response, it is thought to speed up the subsequent processing (i.e., categorization) of similarly valenced stimuli. For example, a prime that elicits negative affect would speed up the categorization of negative words but slow down positive word categorization. By using congruent (e.g., the word "red" in red ink) or incongruent (e.g., the word "red" in blue ink) Stroop stimuli as primes, Dreisbach and Fischer (2012) demonstrated how perceiving incongruent (i.e., conflict-inducing) stimuli can speed up valence categorization of negative, relative to positive, pictures or words (see also Fritz \& Dreisbach, 2013, 2015; Schouppe et al., 2015).

This work demonstrated the value of assessing affective processing after conflict stimuli, as this shines a new light 
on the motivational dimension of cognitive control. However, although these behavioral studies often link back their findings to theories of ACC, this region's involvement in congruency-dependent affective processes has not yet been studied directly. Inspired by these affective priming paradigms, we here used an fMRI study with postconflict affective picture probe presentation as a gauge on the affective concomitants of conflict processing in ACC. In line with the logic of behavioral priming paradigms, it has also been shown that, when a brain region is involved in processing a particular class of stimuli or signals, it will show a diminished response when two signals of the same class occur in succession, compared with when different signals or stimuli are processed-a phenomenon known as repetition suppression (for a review, see GrillSpector, Henson, \& Martin, 2006). Therefore, following Botvinick's (2007) hypothesis that ACC registers conflict as an aversive signal, we can expect that the presentation of negative pictures after incongruent trials and positive pictures after congruent trials will result in diminished ACC activation, because of affect-based repetition suppression. By contrast, "nonmatching" negative pictures after congruent trials and positive pictures after incongruent trials should result in relatively higher ACC activity, because of a lack of a repetition suppression effect.

Interestingly, these predictions are also compatible with recent reinforcement learning models of ACC function that emphasize a more general role for ACC in signaling unexpected (performance) outcomes (Silvetti, Alexander, Verguts, \& Brown, 2014; Alexander \& Brown, 2011; Silvetti, Seurinck, \& Verguts, 2011). For example, because people learn that the more difficult incongruent trials have a lower likelihood of being followed by a correct and fast response, incongruent trials may become associated with a poorer outcome expectancy. Therefore, when interpreting ACC activation as a surprise reaction to the affective content of the picture, participants may plausibly be more surprised to experience positive affect after an incongruent trial or negative affect after a congruent trial because of their differences in outcome expectancies (Alexander \& Brown, 2011; Silvetti et al., 2011).

Although much of the behavioral and computational work discussed above has focused on conflict processing specifically, Botvinick's (2007) hypothesis can easily be extended to other effortful control processes, such as task switching. Therefore, we examined affective picture probe processing after trials (acting as primes) of a conflict task-switching paradigm. These analyses were applied to a subset of participants from a larger (multigroup) data set that we previously reported on in a different context (Braem et al., 2013). None of the imaging analyses between the current and previous articles overlap. In this paradigm, trials could be defined by both their task rule congruency (i.e., a stimulus could require either the same or a different response in both tasks) and whether the current task was the same (i.e., task repetition) or different (i.e., task alternation) to the preceding trial. Besides the well-known observation that people react slower and less accurately on task alternations as opposed to task repetitions (i.e., the task switch cost; Monsell, 2003), task rule congruency effects are also found to be larger on task alternations than task repetitions (Meiran, 2000). The latter observation is assumed to reflect an automatic priming effect from the task rules that were active on the previous trial (Meiran \& Kessler, 2008). Namely, the competing task rules are more active on task alternation than task repetition trials because they were only executed on the preceding trial and therefore interfere more with the required task rules on the present trial (i.e., larger task rule congruency effects).

According to Botvinick (2007), both conflict processing and task switching can be considered effortful processes, characterized by their higher RTs and error rates on incongruent trials and task alternations, respectively. Consistent with their aversive task demands, people will actively avoid tasks that require a higher amount of task switching (Kool, McGuire, Rosen, \& Botvinick, 2010) or conflict processing (Schouppe, Ridderinkhof, Verguts, \& Notebaert, 2014). Hence, task alternations can be considered similar to cognitive conflict in terms of cognitive effort, and therefore, we could expect a similar effect on affective picture processing after task alternations versus task repetitions, as we do after incongruent versus congruent trials. Second, we can expect an enhanced effect of congruency-dependent affective processing in ACC during task alternations-given the observation that congruency effects are enhanced during task alternations.

In summary, our primary goal was to provide a first direct test of the proposal that conflict is registered as an aversive event by ACC, by adopting the logic of the affective priming paradigm from a purely behavioral context (Dreisbach \& Fischer, 2012) to a neuroimaging setting. As a secondary goal, our task protocol also allowed us to explore the affective signature of task switching and how the relationship between conflict processing and affective responses in ACC may be modulated by task switching.

\section{METHODS \\ Participants}

Seventeen participants took part in this study. One participant was not included in the analyses because 30\% of their responses exceeded the response registration deadline. The remaining 16 participants (eight women and eight men, mean age $=27$ years, $S D=7$ years) had a mean of $3.1 \%$ unregistered responses $(S D=3 \%)$. Every participant had normal or corrected-to-normal vision, was right-handed as assessed by the Edinburgh Handedness Inventory, and reported no current or history of neurological, psychiatric, or major medical disorder. Every participant gave their written informed consent before the 
experiment and was paid $\$ 35$ for participating afterward as well as an extra $\$ 16$ as part of the experiment's reinforcement schedule (which will be explained below). The study was completed with the approval of the Duke University Health System institutional review board.

\section{Stimuli}

As affective probe stimuli, we used 50 positive and 50 negative pictures from the International Affective Pictures System (IAPS; Lang, Bradley, \& Cuthbert, 2008) database. The pictures were matched on their semantic content (e.g., cute animal vs. dangerous animal, crying baby vs. smiling baby, sunset vs. thunderstorm) to avoid differences in picture processing that could be attributable to other non-affect-related features (e.g., living vs. nonliving). The imperative task stimuli, which served as "primes" in this affective priming protocol, consisted of the (affectively neutral) numerals 1-9, excluding 5; these stimuli were always presented in isoluminant green or blue. All pictures and task stimuli were centrally presented on a black background. The experiment was projected on a back-projection screen, which participants viewed in a mirror mounted to the head coil. This arrangement resulted in picture sizes of approximately $10^{\circ}$ wide and $7.4^{\circ}$ long and task stimuli of approximately $0.4^{\circ}$ wide and $0.8^{\circ}$ long. Responses were made via an MR-compatible response box (Current Designs, Philadelphia, PA) positioned on the participants' abdomen (perpendicular to the length of their body). Participants had to use their left or right hand to press the leftmost or rightmost button (of four horizontally aligned response buttons), respectively. The stimuli were presented with Presentation software (Neurobehavioral Systems, Berkeley, CA).

\section{Procedure}

To assess the effects of conflict processing during task repetitions or task alternations on affect processing (positive or negative IAPS pictures), we presented an affective picture probe (positive or negative) after each trial (acting as a prime) of a standard conflict task-switching paradigm. Importantly, picture valence was always selected at random and thus independent of the congruency or task-switching conditions. However, whether a picture would be presented (independent of its valence) was dependent on performance speed and accuracy. Specifically, participants were informed that task stimuli would be followed by a randomly chosen positive or negative picture and that positive pictures were associated with 10 cents of monetary gain (to assure picture valence processing), but there would be no picture presentation after incorrect or too slow responses (exceeding the 1500-msec deadline).

During the task, participants had to respond to the magnitude or parity of the task stimulus, depending on its color. For example, when the number was presented in green, participants had to press left when it was smaller than 5 and right when it was larger than 5 . Alternatively, when the number was presented in blue, participants had to respond to its parity by indicating whether it was odd with a left-hand response or even with a righthand response. The color cuing the magnitude versus parity task was counterbalanced across participants. Importantly, each number was associated with one response for each task set. Because these task sets were assigned to overlapping response sets, each number could either produce the same (i.e., congruent) or different (i.e., incongruent) responses in the two tasks. Moreover, the stimuli were presented in a random order (excluding number repetitions), which could result in task repetitions and task alternations. Overall, these manipulations produced a factorial design with three within-participant factors of interest: prime trial congruency (congruent vs. incongruent trial), task sequence (task repetition vs. task switch), and probe valence (positive vs. negative). For the behavioral data analysis, the former two factors allowed us to assess the classic congruency effect, task-switching effect, and the predicted enhancement of congruency effects on task alternations (Meiran, 2000). By contrast, our fMRI analyses exploited the full three-way design by focusing on the neural activity elicited by affective picture probe stimuli as a function of picture valence and the congruency and task sequence status of the preceding prime stimulus.

As can be seen in Figure 1A, each trial started with the presentation of a colored number stimulus $0.6^{\circ}$ above a central fixation dot for $200 \mathrm{msec}$. The central fixation dot remained on-screen throughout the task. Participants were required to respond within $1500 \mathrm{msec}$ after stimulus onset. After a variable stimulus-picture interval, an IAPS picture was presented for $500 \mathrm{msec}$, followed by another variable picture-stimulus interval until the next digit stimulus was presented. Importantly, the stimuluspicture interval was time-locked to the stimulus onset and thus independent of response speed, ensuring that differences in picture processing after different task conditions could not be attributed to differences in the stimulus-picture interval. Both the stimulus-picture and picture-stimulus intervals were independently randomized and drawn from a pseudoexponential distribution $(50 \%$ lasted $3 \mathrm{sec} ; 25 \%, 3.5 \mathrm{sec} ; 12 \%, 4 \mathrm{sec} ; 7 \%, 4.5 \mathrm{sec}$; and $6 \%, 5 \mathrm{sec}$ ), resulting in a mean time interval of $\sim 3.5 \mathrm{sec}$. This way, picture presentation can be studied independently from task stimulus presentations (e.g., Ollinger, Shulman, \& Corbetta, 2001). When the participant's response was incorrect or exceeded the response deadline, picture presentation was replaced by the presentation of the fixation dot for 500 msec.

Participants performed five experimental blocks of 64 trials each during scanning, after a short practice block of 32 trials outside the scanner. In between blocks, there was a short break during which the participants could see their updated score. Each of the 32 stimulus-picture combinations (eight numbers $\times$ two task colors $\times$ picture 


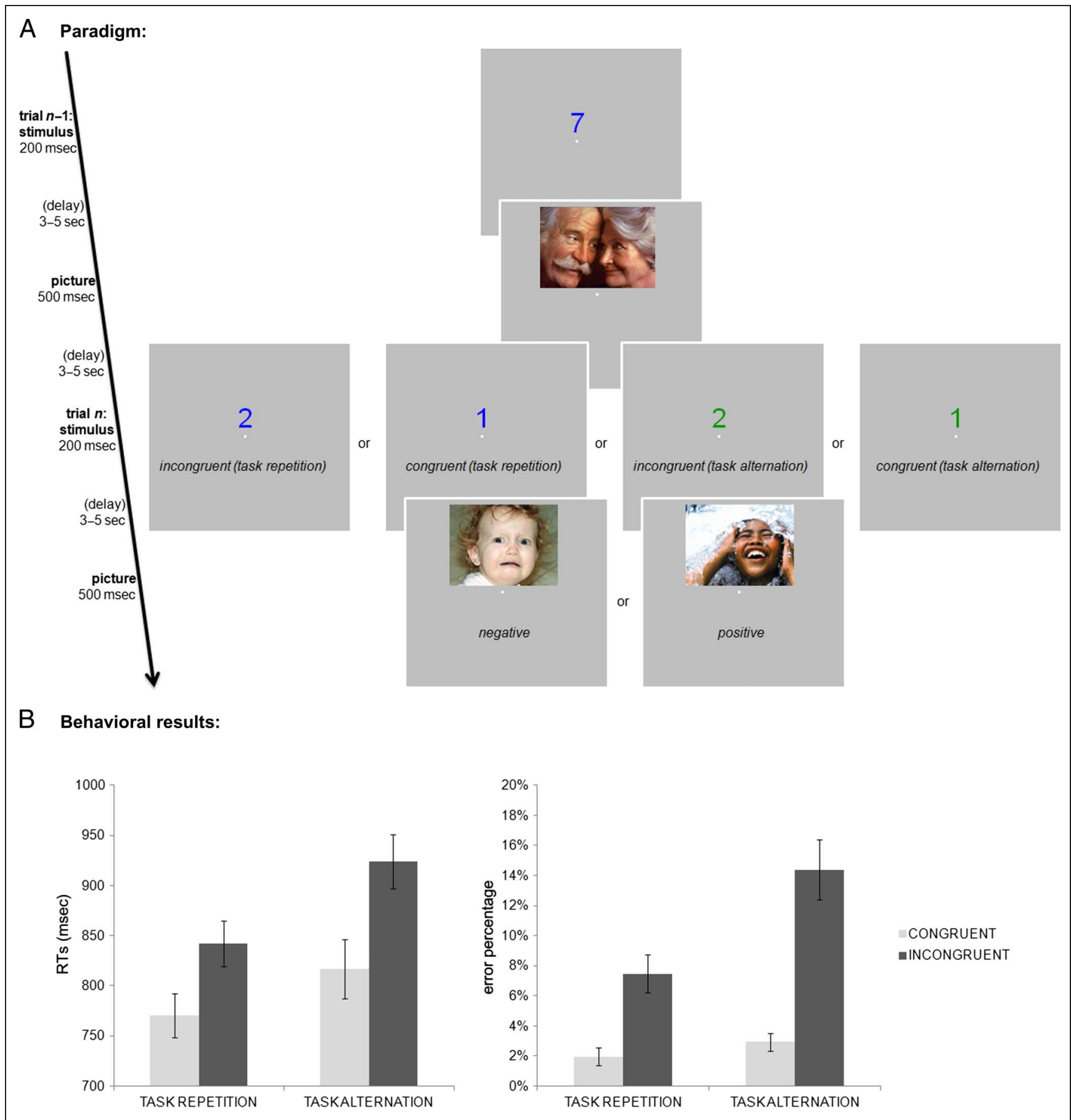

Figure 1. (A) Trial procedure (two successive trials) and different task conditions of interest are denoted. Participants were presented with randomly chosen positive or negative pictures after each task stimulus, except when the preceding response to the task stimulus was incorrect or too slow $(>1500 \mathrm{msec}$ ). Participants had to respond to the digit stimuli's parity or magnitude, depending on stimulus color. Examples of the different possible task conditions of interest are denoted: Picture processing was investigated depending on its valence (negative or positive) and the congruency (incongruent or congruent) and task sequence (repetition or alternation) identity of the immediately preceding stimulus. The words in italic were not presented during the experiment, and the background color in the experiment was black. (B) The behavioral results demonstrate how both RTs and error rates were higher for incongruent relative to congruent trials. Moreover, this difference between both congruency conditions was smaller on task repetitions relative to task alternations. All error bars are \pm 1 SEM.

valence) were presented 10 times in a randomized order. IAPS pictures were randomly chosen from the appropriate valence group but never reoccurred within a block. Although there was some variation in the amount of money participants obtained during the experiment (because of differences in performance accuracy), all participants were given the same maximum amount of money possible at the end of the experiment. 


\section{Behavioral Data Analyses}

All trials after an error and the first trial of each block were removed from the analyses. We carried out an ANOVA with the within-participant factors Trial congruency (congruent vs. incongruent) and Task sequence (repetition vs. switch) on correct RTs and error rates.

\section{fMRI Data Acquisition}

Imaging was conducted on a GE Discovery MR750 system (GE Healthcare Systems, Milwuakee, WI) at 3.0 T using a standard head coil. We acquired functional images parallel to the AC-PC plane with a T2*-weighted single-shot gradient EPI sequence of 36 contiguous axial slices (repetition time $=2000 \mathrm{msec}$, echo time $=28 \mathrm{msec}$, flip angle $=$ $90^{\circ}$, field of view $=192 \mathrm{~mm}$, array size $=64 \times 64$ ) with 3 -mm thickness and $3 \times 3 \mathrm{~mm}$ in-plane resolution. Structural images were acquired with a T1-weighted Fast Spoiled Gradient-Recalled-Echo axial scan using a 3-D inversion recovery prepared sequence, recording 120 slices with 1 -mm thickness and in-plane resolution of $1 \times 1 \mathrm{~mm}$.

\section{fMRI Data Analysis}

The preprocessing steps and statistical analyses were performed using SPM8 (www.fil.ion.ucl.ac.uk/spm/software/ spm8/). Functional data were slice-time corrected and spatially realigned to the first volume of the task. The structural image was normalized to the Montreal Neurological Institute (MNI) template brain (resampled voxel size $=$ $2 \mathrm{~mm}^{3}$ ). These normalization parameters were then applied to the functional images to ensure an anatomically informed normalization. The first five volumes of each run in which no stimulation occurred were discarded before estimating statistical models, and a 128-sec temporal high-pass filter was applied to remove low-frequency drifts. Temporal autocorrelations were estimated using restricted maximum likelihood estimates of variance components with a first-order autoregressive model, and the resulting nonsphericity was used to form maximum likelihood estimates of activations. A spatial smoothing filter of 8-mm FWHM was applied.

Event-related regressors convolved with a canonical hemodynamic response function were created corresponding to the picture onsets and stimulus onsets of each trial. Importantly, the picture-locked regressors were defined by the valence of the picture (positive or negative) as well as the congruency and the task sequence identity of the preceding stimulus (congruent or incongruent and task repetition or task alternation). We also modeled the stimulus-locked regressors as a function of their congruency and task sequence features as well as error trials, trials after an error, and the first trial of each run as separate nuisance variables. Last, both picture- and stimulus-locked regressors were further defined by the picture valence of the preceding trial to control for pos- sible carry-over effects of previous affective picture presentations. Single-participant contrasts on picture-locked regressors were calculated to establish the hemodynamic correlates of picture valence, depending on the preceding congruency and task sequence conditions. Specifically, we first investigated the contrast between negative versus positive picture presentation after congruent versus incongruent trials, for task repetitions only. This contrast allows us to evaluate our main hypothesis that ACC is sensitive to the aversiveness of cognitive conflict (Botvinick, 2007) in a way that is most similar to earlier behavioral studies, which used single-conflict tasks only (for a review, see Dreisbach \& Fischer, 2015). In a second step, we also looked at the picture-locked contrast that includes the factors Picture valence and Task sequence to study the affective evaluation of task-switching conditions. Finally, we analyzed the contrast between picture valence, congruency, and task sequence to further explore the role of task sequences in the hypothesized congruency-dependent affective picture processing of ACC.

To control for false-positive rates, we determined contrast-specific combined voxel activation intensities and cluster extent thresholds that are corrected for multiple comparisons by using $3 d$ ClustSim (afni.nimh.nih. gov/pub/dist/doc/program_help/3dClustSim.html). This software runs 10,000 Monte Carlo simulations that take into account the whole-brain search volume and estimated smoothness of each axis of the respective group SPMs and thereby generates probability estimates of a random field of noise producing a cluster of voxels of a given extent for a set of voxels passing a specific voxelwise $p$ value threshold, which we set at .005 for all analyses. Given this threshold, the $3 d$ ClustSim simulations determined that cluster sizes of 301-334 voxels, depending on the specific contrast, corresponded to a combined threshold of $p<.05$ (corrected).

\section{ROI Analyses}

We extracted mean $\beta$ estimates (i.e., mean cluster activation) from empirically defined ROIs with Marsbar software (marsbar.sourceforge.net/) to evaluate interaction effects.

\section{RESULTS}

\section{Behavioral Data}

Mean accuracy on this task was $89.9 \%$, and mean RT was $834 \mathrm{msec}$. As expected, there was a typical congruency effect that was expressed in higher RTs, $F(1,15)=54.1$, $p<.001$, and higher error rates, $F(1,15)=48.9, p<$ .001 , for incongruent trials (883 msec, $10.9 \%$ ) as opposed to congruent trials (793 msec, $2.4 \%$ ). We also observed a significant task-switch cost in both RTs, $F(1,15)=44.0$, $p<.001$, and error rates, $F(1,15)=28.3, p<.001$, reflecting higher RTs and error rates when a task alternated (870 msec, 8.6\%) as opposed to when the task repeated 
(806 msec, 4.7\%). Furthermore, there was a significant interaction between congruency and task sequence in both RTs, $F(1,15)=7.3, p<.05$, and error rates, $F(1$, $15)=11.7, p<.01$, because of a greater congruency effect for task-switch than task-repeat trials or, conversely, a larger switch effect for incongruent than for congruent trials (see Figure 1B). No other main effects or interactions reached significance (all $p s>.1$ ).

Thus, in line with expectations, these data confirm that incongruent trials were more difficult than congruent trials and task alternations were more demanding than task repetitions. Moreover, consistent with the previous literature, the congruency effect was more pronounced during task alternations than task repetitions (Meiran, 2000). As outlined above, both observations are important in setting up our predictions regarding the influence of conflict conditions on affective processing.

\section{fMRI Data: The Effect of Conflict on Affective Picture Processing}

Next, we analyzed the picture-locked interaction contrast that includes both congruency and picture valence, for task repetitions only. We focused on task repetitions, as these most closely resemble the task conditions under which the affective signatures of conflict processing have previously been investigated (Fritz \& Dreisbach, 2013, 2015; Schouppe et al., 2015; Dreisbach \& Fischer, 2012; Brouillet et al., 2011). If incongruent (relative to congruent) trials are indeed experienced as more negative, because of either their higher control demands (Botvinick, 2007) or lower outcome expectancy (Alexander \& Brown, 2011; Silvetti et al., 2011), we expected these trials to elicit lower ACC activation during subsequent affectively matching negative picture presentations but higher ACC activations during the presentation of affectively nonmatching positive picture presentations and vice versa for congruent trials.
The contrast for the Picture valence $\times$ Congruency interaction on task repetitions resulted in the wholebrain corrected activations of the (dorsal) ACC (Figure 2A) as well as the dorsomedial pFC (dmPFC), left inferior frontal sulcus, left insula, left precuneus, and right thalamus (see Table 1). Consistent with earlier reports of conflict-related ACC activation, our ACC activation was restricted to more dorsal portions of ACC, seemingly extending into the SMA and other more dorsal or prefrontal medial regions. Alternatively, this could also reflect activity in the paracingulate sulcus, a portion of the dorsal ACC that is known to sometimes expand dorsally (as also discussed in this review on ACC function; Shackman et al., 2011). The interaction effect in the activation of ACC was in line with our expectations regarding its role in the affective evaluation of congruency conditions (Alexander \& Brown, 2011; Silvetti et al., 2011; Botvinick, 2007). To determine the source of this interaction, we extracted mean ROI activation values and submitted them to planned comparisons. Specifically, we extracted the mean ROI activation of the cluster activation in ACC/dmPFC (Figure 2C) to evaluate the more anterior cluster. The more posterior ACC cluster, however, was connected to a larger cluster whose peak activation was in the right thalamus (see Table 1). Therefore, this ACC activation was evaluated by analyzing the mean activation around this cluster's third local maximum, which was closest to ACC (a 5-mm sphere around $x=6, y=20, z=$ 38). Besides the significant interaction between Congruency and Valance (on which the ROI was selected), ACC/ dmPFC cluster also showed a main effect of Valence with overall higher activation during negative as opposed to positive picture presentations, $F(1,15)=10.34, p<.01$, replicating a host of previous imaging studies (reviewed in Etkin et al., 2011; Shackman et al., 2011). The more posterior ACC activation did not show this main effect, $F(1,15)=2.09, p=.17$. More importantly, as can be
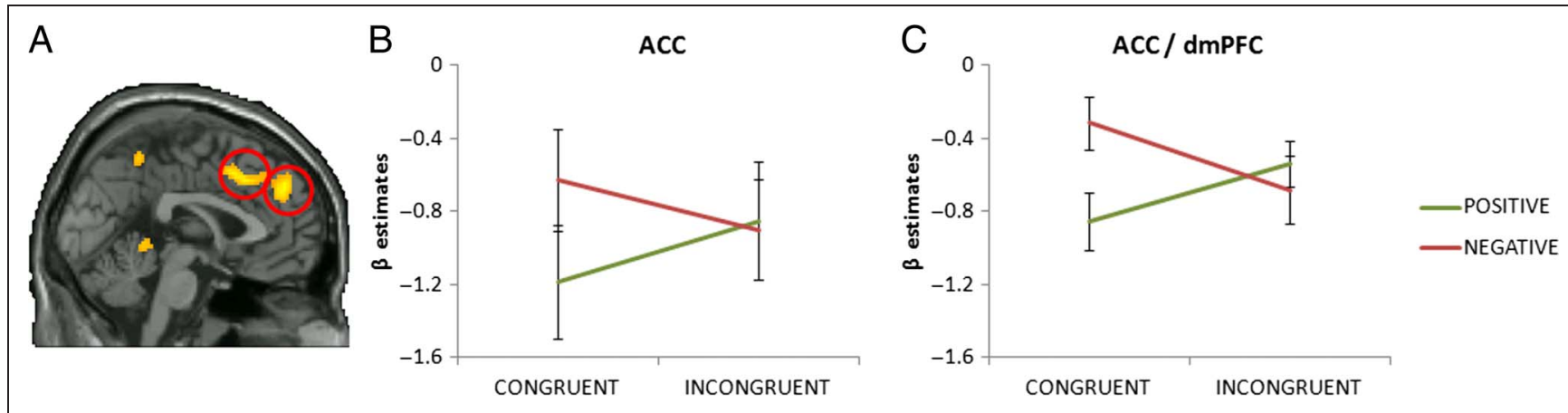

Figure 2. (A) ACC as identified by the picture-locked contrast for the congruency-dependent valence effect on task repetitions (valence effect after congruent trials $>$ valence effect after incongruent trials) regression analysis, plotted at $p<.05$ (corrected) on sagittal $(x=0)$ of an individual brain in MNI space. (B, C) Group mean activations ( $\beta$ estimates \pm SEM) in ACC are plotted for each valence (green is positive; red is negative) and congruency condition, for task repetitions only. The mean activations were based on either the mean cluster activation in ACC/dmPFC (C; abbreviated by ACC/dmPFC) or a 5-mm sphere around a more posterior ACC cluster's peak activation (B; $x=6, y=20, z=38$ ), because this peak was part of a cluster extending into other regions as well (see Results). The error bars are displayed for the purpose of visual presentation (not statistical inference) of the patterns of activation means and variance observed in the ROIs that were identified based on the statistical inference drawn by the whole-brain corrected group analysis. 
Table 1. Picture Onset Locked Activations Revealed by the Picture Valence $\times$ Congruency Condition Contrast for Task Repetitions Only, the Picture Valence $\times$ Task Sequence Contrast for Congruent Trials Only, and the Picture Valence $\times$ Congruency Condition $\times$ Task Sequence Contrast

\begin{tabular}{|c|c|c|c|c|c|}
\hline Anatomical Area & $x$ & $y$ & $z$ & Voxels & $T_{\max }$ \\
\hline \multicolumn{6}{|c|}{$\begin{array}{l}\text { Picture valence as a function of preceding stimulus congruency, on task repetitions only (valence effect after congruent stimuli }> \\
\text { valence effect after incongruent stimuli) }\end{array}$} \\
\hline $\mathrm{ACC} / \mathrm{dmPFC}$ & -6 & 42 & 30 & 618 & 5.18 \\
\hline Left inferior frontal sulcus/left insula & -30 & 6 & 18 & 640 & 5.83 \\
\hline Left superior parietal lobe/left precuneus & -34 & -56 & 58 & 1320 & 4.64 \\
\hline Right thalamus/ACC & 14 & -26 & 16 & 2202 & 5.14 \\
\hline \multicolumn{6}{|c|}{$\begin{array}{l}\text { Picture valence as a function of preceding task sequence, on congruent trials only (valence effect after task repetitions }> \\
\text { valence effect after task alternations) }\end{array}$} \\
\hline ACC/SMA & 4 & 16 & 60 & 800 & 4.95 \\
\hline Cerebellum & 8 & -52 & -6 & 450 & 5.39 \\
\hline \multicolumn{6}{|c|}{ Picture valence as a function of preceding stimulus congruency and task sequence } \\
\hline Dorsal ACC & -2 & 20 & 54 & 362 & 3.94 \\
\hline Left inferior frontal sulcus/junction & -28 & 8 & 44 & 572 & 6.11 \\
\hline Left superior parietal lobe/left precuneus & -40 & -24 & 32 & 5258 & 5.50 \\
\hline Left STS & -38 & -34 & 0 & 979 & 5.39 \\
\hline Thalamus/cerebellum & -4 & -52 & -6 & 1652 & 5.04 \\
\hline Right superior temporal gyrus/hippocampus & 44 & 0 & -26 & 379 & 5.44 \\
\hline
\end{tabular}

seen in Figure $2 \mathrm{~B}$ and $2 \mathrm{C}$, the interaction between congruency and picture valence in ACC/dmPFC originated from a significantly lower activation for negative pictures after incongruent as opposed to congruent trials, $t(15)=$ $2.971, p=.01$, and positive pictures after congruent relative to incongruent trials, $t(15)=3.989, p=.001$. A similar pattern could be observed in the more posterior ACC cluster $(t(15)=2.098, p=.053$, and $t(15)=1.925, p=$ .073 , respectively). These results are concordant with the idea that the higher control demands associated with incongruent, relative to congruent, trials triggered negative evaluation (Botvinick, 2007) that attenuated ACC response to negative, but not positive, picture probes. Alternatively, when interpreting ACC activation as violations of outcome expectancies (Alexander \& Brown, 2011; Silvetti et al., 2011), participants might have been more surprised to encounter negative pictures after a congruent trial or positive pictures after an incongruent trial. Both interpretations are in line with our predictions and the available literature arguing that incongruent trials are experienced as aversive events (Dreisbach \& Fischer, 2015; Inzlicht et al., 2015). Similar to ACC/dmPFC, the interaction in the three other regions (one of which includes the sphere of the more posterior ACC activation) was also driven by a significantly higher activation both for negative pictures after congruent as opposed to incongruent trials, $t \mathrm{~s}(15)>2.890, p \mathrm{~s}<.05$, and positive pictures after incongruent relative to congruent trials, $t \mathrm{~s}(15)>3.054$, $p s<.01$. Different from ACC/dmPFC, however, the other three regions did not show a main effect of Valence, $F(1,15)<1.15, p>.3$.

\section{fMRI Data: The Effect of Task Switching on Affective Picture Processing}

Second, we studied the picture-locked interaction contrast that includes both task sequence conditions and picture valence. The behavioral affective signatures of cognitive conflict are well documented (e.g., Dreisbach \& Fischer, 2012). However, similar automatic affective evaluations of task-switching conditions remain to be tested. Still, although the framework of Botvinick (2007) mainly focuses on conflict processing, its predictions can easily be extended to task switching. Similar to conflict processing, task switching can be considered an effortful mental process that could also register as an aversive signal in ACC. Consistently, people have been found to actively avoid choice decks that are associated to a higher amount of task switching (Kool et al., 2010).

Our first brain analyses did not support this hypothesis. The contrast for Picture valence $\times$ Task sequence interaction did not reveal any significant whole-brain corrected activations. In addition, when testing in the opposite direction, no effects of task-switching conditions on affective picture processing were observed. However, in close analogy to our analysis into the effects of congruency on 
task repetitions only, one could also look at the effects of task switching on congruent trials only, canceling out the arguably interfering or obfuscating effects of incongruent trials. Indeed, the contrast for the Picture valence $\times$ Task sequence interaction on congruent trials only revealed whole-brain corrected activations of the SMA and (dorsal) ACC (Figure 3A) as well as the cerebellum (see Table 1). Similar to the congruency-dependent analysis, follow-up analyses on the mean ROI activation values showed that this ACC/SMA region showed an overall higher activation to negative relative to positive picture presentations, $F(1$, $15)=6.79, p=.02$. Moreover, the interaction between task sequence and picture valence was again driven by a significantly lower activation to both negative pictures after task alternation as opposed to task repetition trials, $t(15)=$ $3.266, p=.005$, and positive pictures after task repetition relative to task alternation trials, $t(15)=2.599, p=.02$ (Figure 3B).

\section{fMRI Data: The Role of Task Sequence in Congruency-dependent Affective Picture Processing}

Next, we turned to task alternations to see if task switching either enhanced or counteracted the affective evaluation of congruency. On task alternations, the congruency effect is enhanced as compared with task repetitions, as evidenced by both the RT and error rate analyses (see Figure 1B). Therefore, if the reported effects of congruency on affect processing are indeed a consequence of their associated cognitive effort (Botvinick, 2007), we would expect these aversive effects to be further enhanced during task alternations.

As a first test of this hypothesis, we reanalyzed the mean ROI activation values from ACC/dmPFC region uncovered by the congruency-dependent valence contrast on task repetitions (Figure 2A) but now focused on its activation pattern during task alternations instead. Intriguingly, in contrast to its modulations during task repetitions, ACC/dmPFC was no longer responsive to picture valence, $F(1,15)=1.376, p>.25$, nor its interaction with congruency, $F(1,15)<1$, during task alternations. Similarly, a separate whole-brain analysis of the Congruency $\times$ Picture valence contrast on task alternations only did not reveal any significant cluster activations (in neither direction). These analyses were further confirmed by a whole-brain analysis of the three-way interaction between task sequence, picture valence, and congruency, which documented activations consistent with these abovementioned results (Figure 4A). Namely, the analysis again uncovered the (dorsal) ACC as well as the left inferior frontal sulcus, left precuneus, left superior parietal areas, the left STS, and right superior temporal gyrus. Although the peak ACC activation in this contrast (Figure 4A) lies a little more posterior $(x=$ $-2, y=20, z=54$ ) than the peak of the cluster displayed in Figure 2A, its activation pattern remained qualitatively unchanged (trend level analyses at corrected $p<$ .1 revealed a more anterior ACC activation as well). Thus, similar to the previous ROI, ACC showed a main effect of Valence, $F(1,15)=8.170, p<.05$. Moreover, the interaction between Task sequence, Picture valence, and Congruency (on which the ROI was selected) was again driven by a congruency-dependent processing of picture valence on task repetitions, $F(1,15)=11.020, p=.005$ (Figure $4 \mathrm{~B})$, but not on task alternations, $F(1,15)=$ $2.870, p>.1$ (Figure $4 \mathrm{C}$ ). Planned comparisons on the task repetition trials again showed lower activation for positive pictures after congruent as opposed to incongruent trials, $t(15)=2.646, p=.018$, and negative pictures after incongruent relative to congruent trials, $t(15)=$ $1.944, p=.071$. The reverse contrast (e.g., regions showing a larger congruency-dependent valence effect on task alternations) did not garner any significant (or trend level) above-threshold clusters. Together, these analyses clearly suggest that the affective priming effect from conflict processing was absent on task alternations. These findings argue against the idea that ACC only signals for increased,
Figure 3. (A) ACC/SMA as identified by the picture-locked contrast for the task sequencedependent valence effect on congruent trials only (valence effect after task repetitions $>$ valence effect after task alternations) regression analysis, plotted at $p<.05$ (corrected) on sagittal $(x=-4)$ of an individual brain in MNI space. (B) Group mean activations $(\beta$ estimates \pm SEM) in ACC/SMA are plotted for each valence (green is positive; red is negative) and task sequence condition, for congruent trials only. The mean activations were based on the mean cluster activation in ACC/SMA. The error bars are displayed for the purpose of visual presentation (not statistical inference) of the patterns of activation means and variance observed in the ROIs that were identified based on the statistical inference drawn by the whole-brain corrected group analysis.
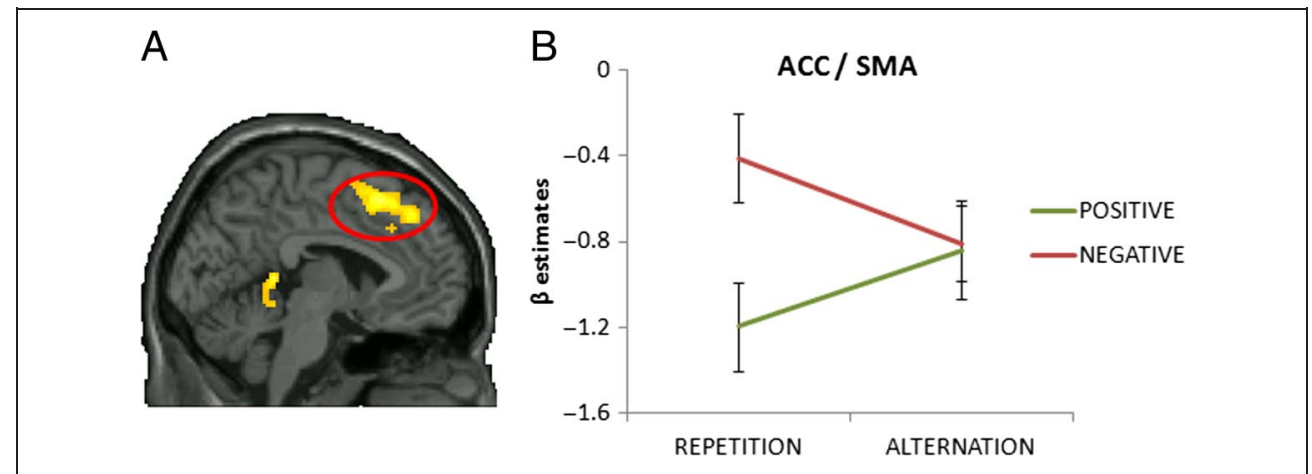

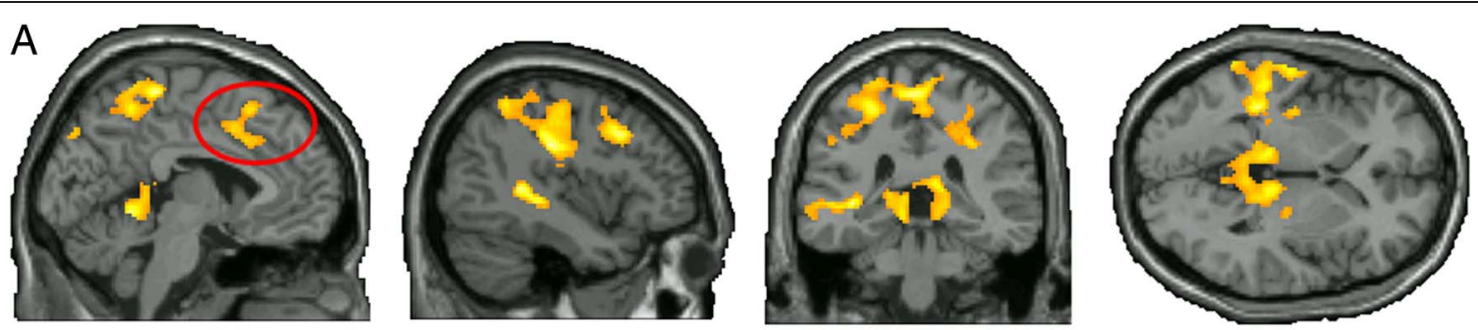

B

Task Repetitions

C

Task Alternations
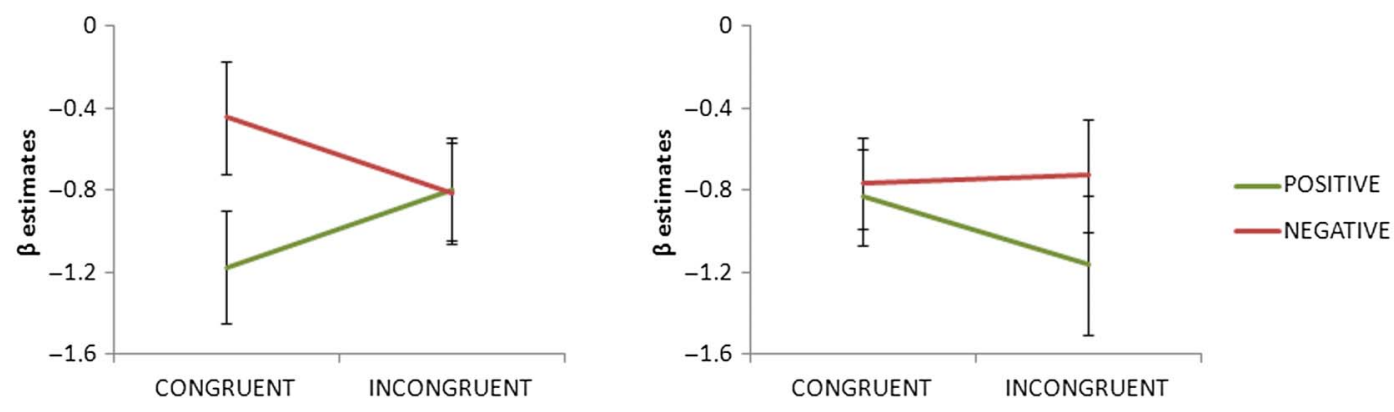

Figure 4. (A) The regions as identified by the picture-locked contrast for the congruency-dependent valence effect on task repetition versus alternation regression analysis, plotted at $p<.05$ (corrected) on sagittal $(x=-4)$, sagittal $(x=-40)$, coronal $(y=-34)$, and axial $(z=0)$ of an individual brain in MNI space. (B, C) Group mean activations ( $\beta$ estimates \pm SEM) in ACC are plotted for each valence (green is positive; red is negative) and congruency, for task repetitions (B) and task alternations (C) separately. The error bars are displayed for the purpose of visual presentation (not statistical inference) of the patterns of activation means and variance observed in the ROIs that were identified based on the statistical inference drawn by the whole-brain corrected group analysis.

inherently negative, cognitive demand (Botvinick, 2007), because based on congruency effects being larger on task alternations, one would have expected their affective consequences to also be enhanced. In hindsight, this can be considered consistent with the reinforcement learning models of ACC function, which stipulate that outcome expectancies are monitored for each task (or context) and congruency condition separately (Alexander \& Brown, 2011; Silvetti et al., 2011). Given that empirical studies have shown that task switching can interfere with task-specific conflict processes (e.g., Braem, Abrahamse, Duthoo, \& Notebaert, 2014; Egner, 2008; Brown, Reynolds, \& Braver, 2007; Goschke, 2000), this raises the possibility that task switching can also disrupt the task-specific evaluation of congruency outcome expectancies, which would lead to a diminished responsiveness of ACC to the affective signatures of conflict processing on switch trials.

\section{DISCUSSION}

In this study, we investigated the effect of different control demands on subsequent affective picture processing. We report for the first time that affective pictures were differentially responded to by ACC dependent on preceding conflict and task-switching conditions. Consistent with the behavioral literature on the affective value of conflict processing (Dreisbach \& Fischer, 2015), we observed diminished ACC activation for negative picture probes after incongruent prime trials or positive picture probes after congruent prime trials. Interestingly, however, this pattern was completely absent on task alternations.

Our main finding is consistent with Botvinick's (2007) hypothesis that ACC responds similarly to cognitive conflict and negative affect, registering both as aversive learning signals that drive certain forms of avoidance learning (see also Shenhav et al., 2013; Shackman et al., 2011). Dreisbach and Fischer (2012) already demonstrated how the presentation of incongruent compared with congruent stimuli can speed up the subsequent evaluative categorization of negative relative to positive pictures (see also Fritz \& Dreisbach, 2013, 2015; Schouppe et al., 2015). We now document the neural correlates of this effect in ACC. Specifically, we show diminished ACC activity in response to negative pictures after incongruent trials (relative to positive pictures and congruent trials), which, following the idea of repetition suppression (Grill-Spector et al., 2006), suggests that ACC processes incongruent trials and negatively valenced picture probe stimuli in a similar fashion (Botvinick, 2007).

Similarly, the diminished processing of positive pictures after congruent relative to incongruent trials could be interpreted as positive affect elicited by congruent conditions. Consistent with the idea that people might take pleasure in encountering a congruent trial, Cannon, Hayes, and Tipper (2010) did report larger zygomaticus activity (muscle activity associated with smiling) after compatible relative to incompatible trials. Therefore, within the local context of an RT experiment, it seems reasonable to 
assume that people can experience some positive emotions during easier trials, in light of knowing that there are more difficult trials too. However, further evidence is required to determine whether congruent conditions automatically elicit positive affect.

Other studies have searched for the processing of a coding of cognitive control "costs" in ACC. For example, McGuire and Botvinick (2010) performed correlation analyses between avoidance ratings or avoidance behavior (in a subsequent test phase) with task-related brain activity during a task-switching study. Although hypothesized, they failed to find a relation between any medial frontal cortex activity and cost processing. However, their analyses focused on block-wise levels of avoidance ratings/ behavior, which might be less sensitive to ACC's coding of costs of moment-to-moment changes in behavior (as also argued by the authors). More indirect evidence can be found in a study by Botvinick, Huffstetler, and McGuire (2009), which demonstrated that ACC's response to effort allocation during task performance inversely predicted the participants' striatal response to subsequent rewards, argued to be consistent with the subjective experience of mental effort. Similarly, Vassena and colleagues (2014) demonstrated how ACC responded more to cues indicating a difficult task or high reward (contingent on task performance), arguably signaling the need for costly and effortful cognitive control processes (see also Prévost, Pessiglione, Météreau, Cléry-Melin, \& Dreher, 2010; Croxson, Walton, O'Reilly, Behrens, \& Rushworth, 2009). These studies show that ACC responds to the cost associated with the allocation of cognitive control resources. However, in our study, we focused on the affective signature of this cost and were able to demonstrate that ACC responds to the affectively aversive signal associated with this allocation of cognitive control.

Importantly, positive pictures were also associated with the prospect of (poststudy) monetary reward. On one hand, this should serve to further enhance the positive affect elicited by the picture stimulus; on the other hand, this means we have to be careful in interpreting the exact source of the putative positive affect. Interestingly, although Shenhav and colleagues (2013) also suggest that cognitive control demands can be considered aversive in nature (as Botvinick, 2007), they added to this hypothesis that the (dorsal) ACC should only be responsive to the value of stimuli that are relevant to the allocation of control. Given that monetary gain can be considered more relevant to the participants' goals (in contrast to the mere valence of the picture), it is possible that the present results are slightly different in nature from the automatic affective evaluation effects in the behavioral studies discussed in the Introduction, where positive stimuli were not paired to monetary gains (e.g., Dreisbach \& Fischer, 2012).

As outlined above, the reinforcement learning models of ACC are also consistent with these results (Silvetti et al., 2011, 2014; Alexander \& Brown, 2011). These models suggest that ACC constantly updates outcome expectancies (e.g., one's chance to respond correctly) for multiple stimulus-response-outcome associations in parallel, thus registering separately for each task and congruency condition whenever outcomes are different than expected. Hence, if we follow this assumption that different congruency conditions automatically activate different outcome expectancies, which are affectively tagged, we can expect the same results as predicted by Botvinick (2007). First, the overall higher activation elicited by negative relative to positive pictures in ACC/ dmPFC can be related to the overall surprise to experience negative affect after a positive outcome expectancy elicited by the correct response (Braem, Coenen, Bombeke, van Bochove, \& Notebaert, 2015; Silvetti et al., 2011, 2014; Desmet, Deschrijver, \& Brass, 2013; Aarts, De Houwer, \& Pourtois, 2012; Wessel, Danielmeier, Morton, \& Ullsperger, 2012; Alexander \& Brown, 2011). However, participants will also (implicitly) have better outcome expectancies on congruent than incongruent trials and thus show higher surprise reactions (i.e., negative prediction errors) whenever a negative event occurs during feedback presentation. Conversely, the enhanced reaction to positive pictures after incongruent relative to congruent trials can be attributed to their lower outcome expectancy and thus higher surprise reaction to this positive outcome (i.e., positive prediction error). In line with this idea, it has been suggested that, in the absence of feedback, the correct response to incongruent versus congruent trials in and of itself could be experienced as more positively surprising or inherently rewarding (Braem et al., 2015; Schouppe et al., 2015; Braem, Verguts, Roggeman, \& Notebaert, 2012).

Our task protocol also facilitated investigating the role of task sequences on the affective evaluation of congruency conditions in ACC. Interestingly, although we did show how task alternations relative to repetitions similarly resulted in a diminished ACC response to negative pictures (on congruent trials only), we also observed that the interaction between congruency and affect processing could only be observed on task repetitions but not task alternations. Although the latter observation was unexpected, it can be considered concordant with the idea that outcome expectancies are task and congruency specific. Previous studies have demonstrated how task switching can disrupt task-specific conflict processing (Brown et al., 2007; Goschke, 2000; for reviews, see Braem et al., 2014; Egner, 2008). Therefore, task switches might have hindered an efficient affective evaluation of congruency conditions. Although this observation seems largely consistent with our interpretation of the reinforcement learning models (Silvetti et al., 2011, 2014; Alexander \& Brown, 2011) of ACC, our experiment cannot, and was not set up to, dissociate between these models and the ideas put forward by Botvinick (2007). We hypothesized that the aversive nature of cognitive demand (Botvinick, 2007) as reflected in the affective evaluation of congruency conditions would be enhanced during task alternationswhich are also thought to be high in cognitive demand 
(Kool et al., 2010) and are known to enhance congruency effects (Meiran, 2000; our data). However, although we hypothesized otherwise, it is still possible that the affective evaluation of cognitive demand is also task specific: Our data can only suggest that the affective concomitants of conflict processing are task specific, irrespective of whether these index the affective evaluation of cognitive demand (Botvinick, 2007) or outcome expectancies (Silvetti et al., 2011, 2014; Alexander \& Brown, 2011).

More generally, this task specificity of conflict processing is also consistent with the observation that adaptations to conflict are often found to be task specific (for reviews, see Braem et al., 2014; Egner, 2008). According to a computational model by Brown et al. (2007), conflict and task alternations evoke opposing learning signals: Whereas the former induces exploitation of task-relevant information, the latter promotes exploration for selecting the appropriate task set. This was recently supported by two independent surprise recognition memory studies demonstrating that memory for task-relevant information was improved under conflict conditions (Krebs, Boehler, De Belder, \& Egner, 2015), whereas it was impaired under task-switching conditions (Richter \& Yeung, 2012). In addition, in studies on affective modulations of cognitive control, a distinction is often made between both types of cognitive effort as they are differentially affected by valence manipulations. Whereas task-switching performance has frequently been reported as improved after positive mood induction (e.g., Dreisbach \& Goschke, 2004), conflict processing has often been found to be impaired under positive mood (e.g., van Steenbergen, Band, \& Hommel, 2010). These findings further suggest that cognitive conflict and task switches elicit opposing processes or strategies and, on a more general level, are in line with our findings that both differentially influenced affective feedback processing.

Notably, we were able to demonstrate the affective signatures of both control processes-cognitive conflict and task switching - but only after canceling out the other (i.e., focus on task repetition trials or congruent trials only, respectively). Importantly, in this study, both factors were manipulated orthogonally. Therefore, participants were required to both switch back and forth between different task sets, while simultaneously shielding the task-relevant information from conflicting stimulus-response associations. This way, both processes, thought to reflect opposing forces of cognitive control (Brown et al., 2007), might have been in constant competition. If we had used two separate designs instead (i.e., a conflict task without task-switching conditions and a task-switching study without conflicting stimuli), we might have been able to find a similar effect on affective picture processing without having to exclude task alternation or incongruent trials. Future studies, including behavioral, are necessary to further investigate the automatic affective evaluation of congruency and task-switching conditions.

Lastly, the present findings also fit with recent observations that emotional distraction by negative pictures (rel- ative to neutral pictures) appears to be reduced after incongruent trials (Cohen, Moyal, \& Henik, 2015; Cohen, Henik, \& Moyal, 2012; Cohen, Henik, \& Mor, 2011; for a review, see Okon-Singer, Lichtenstein-Vidne, \& Cohen, 2013), concordant with our findings that incongruent stimuli reduce the subsequent neural response to negative pictures, which could similarly suggest reduced negative emotion processing after incongruent trials (although note that we lack behavioral measurements to support this claim). For example, Cohen and colleagues (2015) demonstrated how both the pupillary light reflex and the pupil dilation response to negative pictures were attenuated after incongruent trials. The authors interpreted these findings as evidence for the hypothesis that emotional processing is reduced after the recruitment of executive control (Cohen et al., in press; Okon-Singer et al., 2013). Interestingly, as stated in the discussion of their findings (Cohen et al., 2015), this hypothesis would also predict reduced sensitivity to positive picture processing. The present results document a reduced response to negative, but enhanced response to positive, pictures after incongruent trials (see also Fritz \& Dreisbach, 2013, 2015; Schouppe et al., 2015; Dreisbach \& Fischer, 2012). Therefore, it remains to be determined whether studies would find reduced emotional distraction when using positive pictures (as the reduced distraction reported in Cohen et al., 2011, 2012, 2015) or whether such studies would show the opposite pattern instead. The latter would suggest that the results of Cohen and colleagues (2011, 2012, 2015) could have been influenced by the aversive nature of conflict processing (Botvinick, 2007).

In conclusion, our results demonstrated how congruency conditions influence affective processing in ACC. Although ACC responded less to negative pictures after incongruent than congruent trials, it showed more activation to positive pictures after incongruent compared with congruent trials. These results not only add to the growing number of observations that conflict is aversive (for a review, see Dreisbach \& Fischer, 2015) but crucially demonstrate for the first time that ACC can indeed be considered a prime candidate for registering the affective signature of conflict processing, consistent with recent models that emphasize its integrative role in cognition and emotion (Silvetti et al., 2011, 2014; Alexander \& Brown, 2011; Botvinick, 2007). Future studies should extend these findings to other conflict paradigms to determine the robustness and generalizability of our results. Lastly, we demonstrated how this automatic affective evaluation of conflict could be observed on task repetitions only, suggesting that the affective concomitants of cognitive conflicts are processed in a task-specific manner.

\section{Acknowledgments}

S. B. was supported by FWO-Research Foundation Flanders (FWO15/PDO/029). This research was funded by the National Institute of Mental Health Award R01MH087610 (T. E.) and FWO Travel Grant V418212N (S. B.). 
Reprint requests should be sent to Senne Braem, Department of Experimental Psychology, Henri Dunantlaan 2, 9000 Ghent, Belgium, or via e-mail: Senne.Braem@Ugent.be.

\section{REFERENCES}

Aarts, K., De Houwer, J., \& Pourtois, G. (2012). Evidence for the automatic evaluation of self-generated actions. Cognition, 124, 117-127.

Alexander, W. H., \& Brown, J. W. (2011). Medial prefrontal cortex as an action-outcome predictor. Nature Neuroscience, 14, 1338-1344.

Botvinick, M. M. (2007). Conflict monitoring and decision making: Reconciling two perspectives on anterior cingulate function. Cognitive, Affective \& Behavioral Neuroscience, 7, 356-366.

Botvinick, M. M., Braver, T. S., Barch, D. M., Carter, C. S., \& Cohen, J. D. (2001). Conflict monitoring and cognitive control. Psychological Review, 108, 624-652.

Botvinick, M. M., Cohen, J. D., \& Carter, C. S. (2004). Conflict monitoring and anterior cingulate cortex: An update. Trends in Cognitive Sciences, 8, 539-546.

Botvinick, M. M., Huffstetler, S., \& McGuire, J. T. (2009). Effort discounting in human nucleus accumbens. Cognitive, Affective \& Behavioral Neuroscience, 9, 16-27.

Braem, S., Abrahamse, E. L., Duthoo, W., \& Notebaert, W. (2014). What determines the specificity of conflict adaptation? A review, critical analysis, and proposed synthesis. Frontiers in Psychology, 5, 1134.

Braem, S., Coenen, E., Bombeke, K., van Bochove, M. E., \& Notebaert, W. (2015). Open your eyes for prediction errors. Cognitive, Affective \& Behavioral Neuroscience, 15, 374-380.

Braem, S., King, J. A., Korb, F. M., Krebs, R. M., Notebaert, W., \& Egner, T. (2013). Affective modulation of cognitive control is determined by performance-contingency and mediated by ventromedial prefrontal and cingulate cortex. Journal of Neuroscience, 33, 16961-16970.

Braem, S., Verguts, T., Roggeman, C., \& Notebaert, W. (2012). Reward modulates adaptations to conflict. Cognition, 125, 324-332.

Brouillet, T., Ferrier, L. P., Grosselin, A., \& Brouillet, D. (2011). Action compatibility effects are hedonically marked and have incidental consequences on affective judgment. Emotion, 11, 1202-1205.

Brown, J. W., Reynolds, J. R., \& Braver, T. S. (2007). A computational model of fractioned conflict-control mechanisms in task-switching. Cognitive Psychology, 55, 37-85.

Cannon, P. R., Hayes, A. E., \& Tipper, S. P. (2010). Sensorimotor fluency influences affect: Evidence from electromyography. Cognition E Emotion, 24, 681-691.

Cohen, N., Henik, A., \& Mor, N. (2011). Can emotion modulate attention? Evidence for reciprocal links in the attentional network test. Experimental Psychology, 58, 171-179.

Cohen, N., Henik, A., \& Moyal, N. (2012). Executive control attenuates emotional effects-For high reappraisers only? Emotion, 12, 970-979.

Cohen, N., Margulies, D. S., Ashkenazi, S., Schaefer, A., Taubert, M., Henik, A., et al. (in press). Using executive control training to suppress amygdala reactivity to aversive information. Neuroimage, 125, 1022-1031.

Cohen, N., Moyal, N., \& Henik, A. (2015). Executive control suppresses pupillary responses to aversive stimuli. Biological Psychology, 112, 1-11.

Croxson, P. L., Walton, M. E., O'Reilly, J. X., Behrens, T. E., \& Rushworth, M. F. (2009). Effort-based cost-benefit valuation and the human brain. Journal of Neuroscience, 29, 4531-4541.

Desmet, C., Deschrijver, E., \& Brass, M. (2013). How social is error observation? The neural mechanisms underlying the observation of human and machine errors. Social Cognitive and Affective Neuroscience, 9, 427-435.

Dignath, D., \& Eder, A. B. (2015). Stimulus conflict triggers behavioral avoidance. Cognitive, Affective \& Behavioral Neuroscience, 15, 822-836.

Dreisbach, G., \& Fischer, R. (2012). Conflicts as aversive signals. Brain and Cognition, 78, 94-98.

Dreisbach, G., \& Fischer, R. (2015). Conflicts as aversive signals for control adaptation. Current Directions in Psychological Science, 24, 255-260.

Dreisbach, G., \& Goschke, T. (2004). How positive affect modulates cognitive control: Reduced perseveration at the cost of increased distractibility. Journal of Experimental Psychology: Learning, Memory, and Cognition, 30, 343-353.

Egner, T. (2008). Multiple conflict-driven control mechanisms in the human brain. Trends in Cognitive Sciences, 12, 374-380.

Etkin, A., Egner, T., \& Kalisch, R. (2011). Emotional processing in anterior cingulate and medial prefrontal cortex. Trends in Cognitive Sciences, 15, 85-93.

Fazio, R. H. (2001). On the automatic activation of associated evaluations: An overview. Cognition E Emotion, 15, 115-141.

Fritz, J., \& Dreisbach, G. (2013). Conflicts as aversive signals: Conflict priming increases negative judgments for neutral stimuli. Cognitive, Affective \& Behavioral Neuroscience, 13, 311-317.

Fritz, J., \& Dreisbach, G. (2015). The time course of the aversive conflict signal. Experimental Psychology, 62, 30-39.

Goschke, T. (2000). I A intentional reconfiguration and J-TI involuntary persistence in task set switching. In S. Monsell \& J. Driver (Eds.), Control of cognitive processes: Attention and performance XVIII (pp. 331-355). Cambridge, MA: MIT Press.

Grill-Spector, K., Henson, R., \& Martin, A. (2006). Repetition and the brain: Neural models of stimulus-specific effects. Trends in Cognitive Sciences, 10, 14-23.

Inzlicht, M., Bartholow, B. D., \& Hirsh, J. B. (2015). Emotional foundations of cognitive control. Trends in Cognitive Sciences, 19, 126-132.

Kool, W., McGuire, J. T., Rosen, Z. B., \& Botvinick, M. M. (2010). Decision making and the avoidance of cognitive demand. Journal of Experimental Psychology: General, 139, 665-682.

Krebs, R. M., Boehler, C. N., De Belder, M., \& Egner, T. (2015). Neural conflict-control mechanisms improve memory for target stimuli. Cerebral Cortex, 25, 833-843.

Lang, P. J., Bradley, M. M., \& Cuthbert, B. N. (2008). International Affective Picture System (IAPS): Affective ratings of pictures and instruction manual (Technical report A-8). Gainesville, FL: University of Florida.

McGuire, J. T., \& Botvinick, M. M. (2010). Prefrontal cortex, cognitive control, and the registration of decision costs. Proceedings of the National Academy of Sciences, U.S.A. 107, 7922-7926.

Meiran, N. (2000). Reconfiguration of stimulus task sets and response task sets during task switching. In S. Monsell \& J. Driver (Eds.), Control of cognitive processes: Attention and performance XVIII (pp. 377-399). Cambridge, MA: MIT Press.

Meiran, N., \& Kessler, Y. (2008). The task rule congruency effect in task switching reflects activated long-term memory. Journal of Experimental Psychology: Human Perception and Performance, 34, 137-157.

Monsell, S. (2003). Task switching. Trends in Cognitive Sciences, 7, 134-140.

Okon-Singer, H., Lichtenstein-Vidne, L., \& Cohen, N. (2013). Dynamic modulation of emotional processing. Biological Psychology, 92, 480-491. 
Ollinger, J. M., Shulman, G. L., \& Corbetta, M. (2001). Separating processes within a trial in event-related functional MRI: I. The method. Neuroimage, 13, 210-217.

Prévost, C., Pessiglione, M., Météreau, E., Cléry-Melin, M. L., \& Dreher, J. C. (2010). Separate valuation subsystems for delay and effort decision costs. Journal of Neuroscience, 30, 14080-14090.

Richter, F. R., \& Yeung, N. (2012). Memory and cognitive control in task switching. Psychological Science, 23, 1256-1263.

Ridderinkhof, K. R., Ullsperger, M., Crone, E. A., \& Nieuwenhuis, S. (2004). The role of the medial frontal cortex in cognitive control. Science, 306, 443-447.

Schouppe, N., Braem, S., De Houwer, J., Silvetti, M., Verguts, T., Ridderinkhof, K. R., et al. (2015). No pain, no gain: The affective valence of congruency conditions changes following a successful response. Cognitive, Affective \& Behavioral Neuroscience, 15, 251-261.

Schouppe, N., De Houwer, J., Richard Ridderinkhof, K., \& Notebaert, W. (2012). Conflict: Run! Reduced Stroop interference with avoidance responses. Quarterly Journal of Experimental Psychology, 65, 1052-1058.

Schouppe, N., Ridderinkhof, K. R., Verguts, T., \& Notebaert, W. (2014). Context-specific control and context selection in conflict tasks. Acta Psychologica, 146, 63-66.

Shackman, A. J., Salomons, T. V., Slagter, H. A., Fox, A. S., Winter, J. J., \& Davidson, R. J. (2011). The integration of negative affect, pain and cognitive control in the cingulate cortex. Nature Reviews Neuroscience, 12, 154-167.
Shenhav, A., Botvinick, M. M., \& Cohen, J. D. (2013). The expected value of control: An integrative theory of anterior cingulate cortex function. Neuron, 79, 217-240.

Silvetti, M., Alexander, W., Verguts, T., \& Brown, J. W. (2014). From conflict management to reward-based decision making: Actors and critics in primate medial frontal cortex. Neuroscience \& Biobehavioral Reviews, 46, 44-57.

Silvetti, M., Seurinck, R., \& Verguts, T. (2011). Value and prediction error in medial frontal cortex: Integrating the single-unit and systems levels of analysis. Frontiers in Human Neuroscience, 5,75 .

Stroop, J. R. (1935). Studies of interference in serial verbal reactions. Journal of Experimental Psychology, 18, 643-662.

Tracey, I., \& Mantyh, P. W. (2007). The cerebral signature for pain perception and its modulation. Neuron, 55, 377-391.

van Steenbergen, H. (2015). Affective modulation of cognitive control: A biobehavioral perspective. In G. Gendolla, M. Tops, \& S. Koole (Eds.), Handbook of biobehavioral approaches to self-regulation (pp. 89-107). New York: Springer.

van Steenbergen, H., Band, G. P., \& Hommel, B. (2010). In the mood for adaptation how affect regulates conflict-driven control. Psychological Science, 21, 1629-1634.

Vassena, E., Silvetti, M., Boehler, C. N., Achten, E., Fias, W., \& Verguts, T. (2014). Overlapping neural systems represent cognitive effort and reward anticipation. PLoS One, 9, e91008.

Wessel, J. R., Danielmeier, C., Morton, J. B., \& Ullsperger, M. (2012). Surprise and error: Common neuronal architecture for the processing of errors and novelty. Journal of Neuroscience, 32, 7528-7537. 